\title{
Managing Complications in Abdominoplasty: A Literature Review
}

\author{
Pedro Vidal ${ }^{1,2}$, Juan Enrique Berner ${ }^{3,4}$, Patrick A. Will ${ }^{5,6}$ \\ ${ }^{1}$ Clínica La Parva, Santiago; ${ }^{2}$ Universidad San Sebastián, Santiago, Chile; ${ }^{3}$ Department of Plastic Surgery, North Bristol NHS Trust, Bristol; \\ ${ }^{4}$ Kellogg College, University of Oxford, Oxford, UK; ${ }^{5}$ Pastic Surgery Department, BGU Klinik Ludwigshafen, Ludwigshafen; ${ }^{6}$ Ruprecht- \\ Karls-Universität Heidelberg, Heidelberg, Germany
}

Background Abdominoplasty, with or without liposuction, is among the most frequently performed aesthetic procedures. Its main objective is to improve the body contour by means of excising redundant skin and fat tissue. Although abdominoplasty is considered a safe procedure with high satisfaction rates, intraoperative and postoperative complications can become a challenge for the surgical team. The aim of this article is to offer a synopsis of the most common complications arising after abdominoplasty, along with evidence-based guidelines about how to prevent and treat them.

Methods A systematic MEDLINE search strategy was designed using appropriate Medical Subject Headings (MeSH) terms, and references were scanned for further relevant articles. Results According to the published case series, local complications are considerably more common than complications with systemic repercussions. Approximately 10\% to $20 \%$ of patients suffer a local complication following abdominoplasty, while fewer than $1 \%$ suffer a systemic complication. Prevention and management strategies are critically discussed for complications including seroma, haematoma, infection, skin necrosis, suture extrusions, hypertrophic scars, neurological symptoms, umbilical anomalies, deep venous thrombosis and pulmonary thromboembolism, respiratory distress, and death.

Conclusions The complications of abdominoplasty vary in severity and in the impact they have on the aesthetic outcomes. Recommendations for prevention and management are based on various levels of evidence, with a risk of observer bias. However, most complications can be treated appropriately following the current standards, with satisfactory results.

Keywords Abdominoplasty / Lipectomy / Surgery, plastic / Complications / Cosmetic techniques / Reconstructive surgical procedure
Correspondence: Pedro Vidal Clínica La Parva, Av. Las Condes 13.305, Santiago, Chile Tel: +56-2-2207-1125

E-mail: pvidalg@mac.com

This article was presented at the International Society of Aesthetic Plastic Surgery (ISAPS) Course \& The 6th Asian Symposium for Breast Plastic and Reconstructive Surgery (ASBPRS) on 2014, Oct 19, in Bali, Indonesia.

Received: 20 Jan 2017 • Revised: 1 Jun 2017 - Accepted: 13 Jun 2017

pISSN: 2234-6163・ elSSN: 2234-6171・https://doi.org/10.5999/aps.2017.44.5.457• Arch Plast Surg 2017;44:457-468

No potential conflict of interest relevant to this article was reported.

\section{INTRODUCTION}

The abdominoplasty is one of the most commonly performed aesthetic surgical procedures across the world. It is estimated that more than 800,000 people undergo this operation each year, making it the sixth most common cosmetic procedure [1]. The main objective of an abdominoplasty is to reshape the body contour by means of excising redundant skin and fat tissue to remodel the abdominal wall. Since its initial conception more than a century ago, various surgical alternatives have been pro- 
posed [2]. However, it was during the 1960s and 1970s that the contributions of Vernon [3], Pitanguy [4], and Grazer [5] established the founding pillars of modern abdominoplasty.

The contemporary techniques that have subsequently been described share 3 characteristics: limited dissection of the abdominal flap, plication of the rectus abdominis fascia, and resection of a segment of skin and underlying subdermal tissue down to the Scarpa fascia. Performing adjuvant liposuction of the abdominal wall as is a more recent proposal [6] that has led to higher satisfaction rates in select groups of patients [7]. Despite the initial concerns regarding a higher risk of flap necrosis with this technique, since the publication of the studies by Saldanha et al. [8], it has now become a common procedure. A safe approach involves raising a narrow tunnel from the umbilicus up to the xyphoid process, preserving the lateral perforating vessels. The combination of abdominoplasty and liposuction is usually referred to as lipoabdominoplasty.

As is the case for any other surgical intervention, both abdominoplasties and lipoabdominoplasties are susceptible to complications. In 2004, Matarasso et al. [9] surveyed 497 members of the American Society of Plastic Surgeons about their preferences regarding different abdominoplasty techniques and their most frequent complications. The analysis comprised 20,029 procedures, of which 55\% were traditional abdominoplasties, $35 \%$ were lipoabdominoplasties, and $10 \%$ were limited abdominoplasties, also known as mini-abdominoplasties. The local complication rates were $20 \%, 10.3 \%$, and $13.5 \%$, respectively. The systemic complication rate was less than $0.1 \%$ for all techniques.

Every complication poses a challenge for the plastic surgeon and puts the patient-doctor relationship at risk. This has a special relevance for aesthetic procedures, because these are generally elective procedures undergone by healthy patients whose expectations can be ruined by a subsequent complication.

\section{METHODS}

A systematic search strategy for the MEDLINE database was designed using the following Medical Subject Headings (MeSH) terms: "Abdominoplasty/adverse effects," "Intraoperative Complications," and "Postoperative Complications." Original articles and case reports published in English and Spanish were reviewed by 2 authors prior to inclusion in the study. References were screened and relevant articles not found using our research strategy were also included. No exclusion criteria were applied in order to avoid excluding relevant articles.

The most frequent abdominoplasty complications, along with a critical analysis of prevention strategies and management op- tions, are discussed in this article. The level of evidence evaluation for therapeutic studies was carried out following the Oxford Centre for Evidence-Based Medicine recommendations [10].

\section{RESULTS}

A total of 119 articles were found, and 91 were considered to be relevant for this review. The results are presented in a narrative way, separating each specific complication with its risk factors, prevention strategies, and treatment.

\section{Seroma}

The accumulation of serous fluid underneath the abdominal flap is the most frequent complication following an abdominoplasty. In the series of 1,008 cases published by Neaman et al. [11] in 2013, the reported seroma rate was $15.4 \%$. In this study, the authors identified an association between adjuvant liposuction and a higher risk of suffering a seroma, particularly in male patients.

The possibility of a higher incidence of seroma in lipoabdominoplasties than in abdominoplasties without liposuction remains controversial. Najera et al. [12] published a series of 200 patients, showing that the seroma rates in the abdominoplasty and lipoabdominoplasty groups were $16 \%$ and $31.2 \%$, respectively. These percentages are far greater than the $0.04 \%$ seroma rate reported by Hurvitz et al. [13]. Unfortunately, no consensus exists regarding the definition of a clinically significant seroma or an objective method of assessing this outcome.

Different surgical strategies have been proposed to reduce the risk of seroma formation. One of them is the preservation of the Scarpa fascia while raising the abdominal flap, leaving it attached to the rectus abdominis and external oblique muscle fascia. According to studies by Costa-Ferreira et al. [14] and Fang [15], this technique would allow earlier drain removal while avoiding up to $86 \%$ of expected seromas (Level of Evidence: Therapeutic, II). However, some authors have expressed scepticism regarding the impact of a more superficial dissection, especially because of the lack of blinding in the previously discussed studies. Swanson [16] argued that a seroma rate of $5.4 \%$ can be achieved with scalpel dissection below the Scarpa fascia (Level of Evidence: Therapeutic, V). In 2015, Marsh et al. [17] published a prospective randomized study comparing scalpel and handheld electrocautery dissection, finding no difference in the seroma rate between the 2 groups (Level of Evidence: Therapeutic, II).

Another proposed alternative is to use sutures to obliterate the dead space between the Scarpa fascia and the rectus abdominis and external oblique muscle fascia. Baroudi and Ferreira [18], two of this technique's most renowned advocates, have argued 
that collapsing this space reduces the possibility of liquid accumulation. In 2012, Pollock and Pollock [19] published their experience with 597 patients, in whom progressive tension sutures were used for this purpose, reporting only 1 case of seroma in 12 years (Level of Evidence: Therapeutic, IV). In a similar manner, fibrin glue has also been proposed for collapsing the space below the abdominal flap (Level of Evidence: Therapeutic, III [20]) [21].

Fortunately, most seromas usually resolve after puncture and repeated aspiration (Fig. 1). Injection of steroids after draining a seroma to accelerate the process is not backed by supporting evidence. Alkylating agents, such as bleomycin, doxycycline, and talcum powder [22], have been used for recurrent seromas, inspired by their use for persistent pleural effusion. Surgery is a last resort, with the objective of obliterating the space occupied by the seroma by approximating its walls (Level of Evidence: Therapeutic, III [23]) [24].

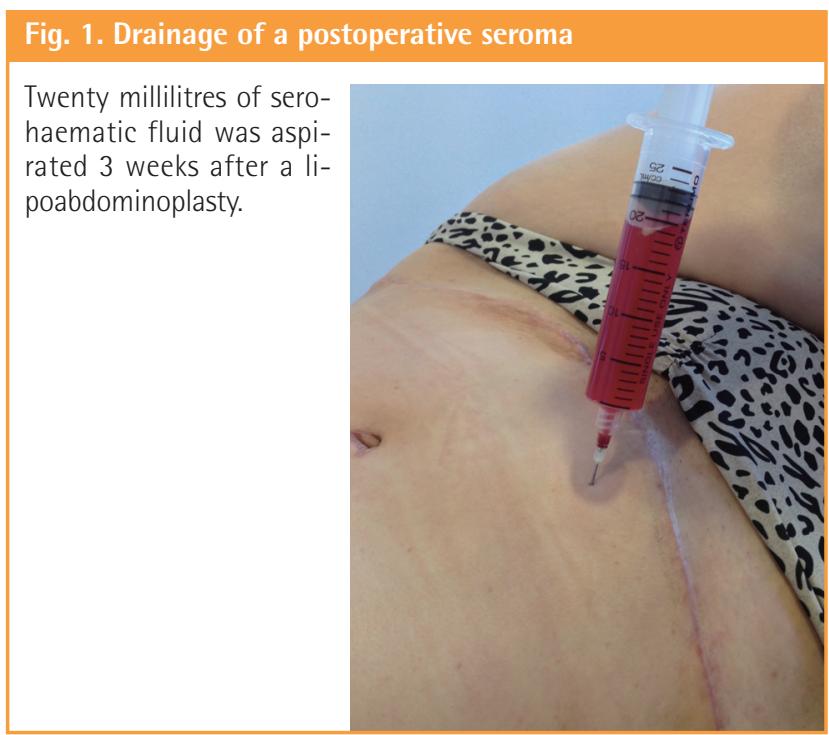

\section{Infection}

Infections are the second most common complication following abdominoplasty, with an estimated incidence between $1 \%$ and 3.8\% [9,11], including operative site infections and infected seromas. There is often inflammation of a delimitated area that typically presents erythema, oedema, tenderness, and an elevated local temperature (Fig. 2). Exudate and systemic symptoms might also be present in more severe infections.

Immunosuppressed states, malnutrition, and diabetes are known risk factors for any kind of infectious process. Particularly, for abdominoplasties, obese and overweight patients seem to have an elevated infection risk (Level of Evidence: Prognosis, IV [25]) [26].

Tobacco consumption also increases the risk of infection, raising the infection rate to $12.7 \%$, compared with $5 \%$ in nonsmokers, according to the case series published by Manassa et al. [27] (Level of Evidence: Prognosis, IV). This is explained by the vasoconstriction that follows smoking, which impairs cellular immunity [28]. Other complications, such as flap necrosis due to insufficient irrigation and seromas, also increase the risk of infection.

Regarding the surgical technique, Samra et al. [29] were not able to retrospectively prove a statistically significant difference in the infection rate between patients who underwent an abdominoplasty and those who underwent an lipoabdominoplasty (Level of Evidence: Prognosis, IV).

Skin bacterial flora accounts for the majority of infections after abdominoplasties, especially Staphylococcus epidermidis, Streptococcus pyogenes, and S. aureus, requiring second-line antibiotic therapy when these species present methicillin resistance [30]. In our experience, we have found a considerable number of Escherichia coli and Enterococcus faecalis infections, probably explained by poor hand hygiene in some patients (Fig. 3). There

\section{Fig. 2. Infected seroma in a 2-week-old lipoabdominoplasty patient}

(A-C) Cellulitis caused by an infected seroma 2 weeks postoperatively. Eighty millilitres of purulent fluid was obtained.
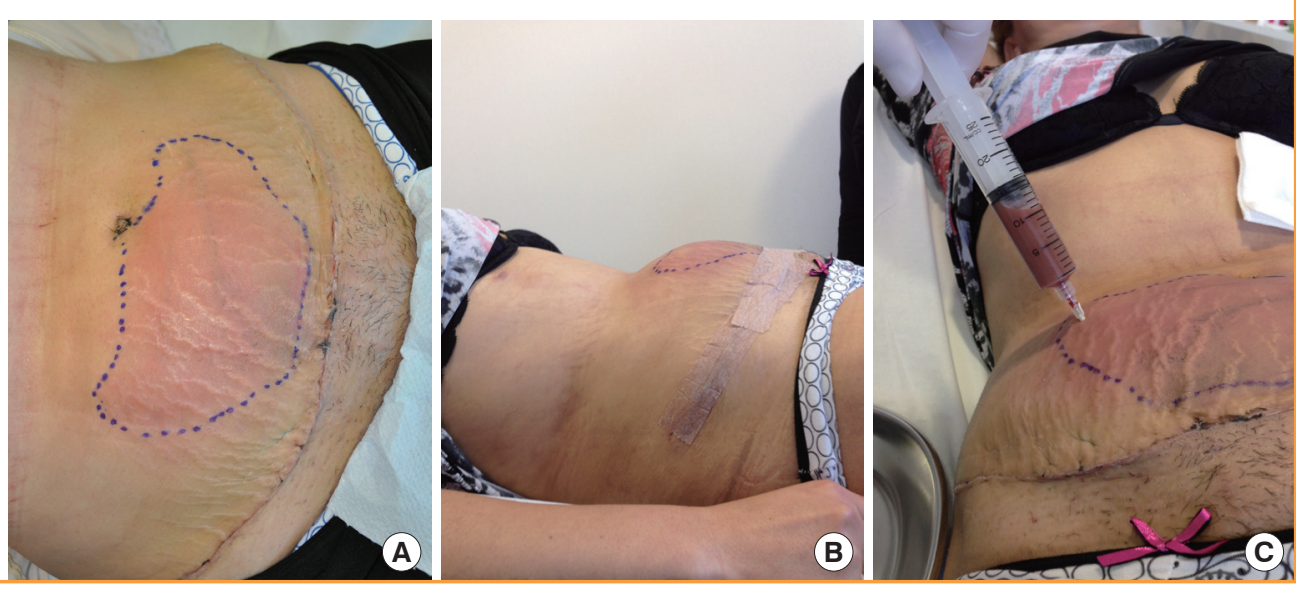
have been isolated reports of infections caused by atypical mycobacteria $[31,32]$, usually in tropical environments.

There is no current consensus on the correct antibiotic prophylaxis that patients should receive prior to abdominoplasty. In 2007, Sevin et al. [33] published a prospective study in which 207 patients were recruited in 3 cohorts: no antibiotic prophylaxis, a single dose of prophylaxis prior to incision, and a preoperative and postoperative prophylaxis scheme. They reported that a single-dose strategy reduced the infection rate from $13 \%$ to $4 \%$, with no added benefit shown in the preoperative and postoperative prophylaxis group (Level of Evidence: Therapeutic, III [33]). Casaer et al. [34] reported an $8 \%$ infection rate in a 300-patient case series with no antibiotic prophylaxis at all (Level of Evidence: Therapeutic, IV).

Antibiotic therapy should always be tailored according to local protocols and cultures. There is generally a quick response to

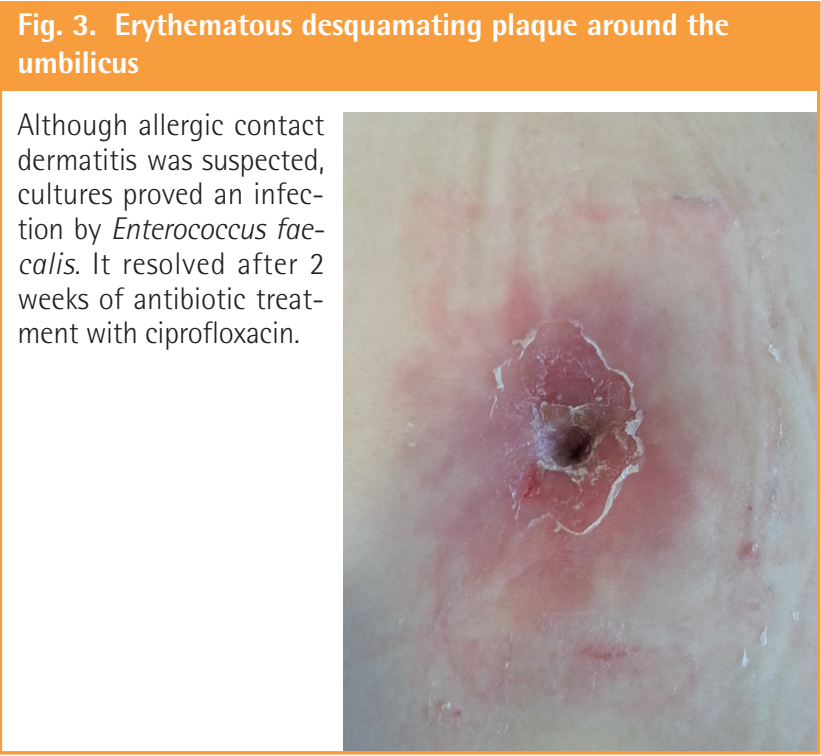

treatment. Reports of severe infections evolving into necrotizing fasciitis, sepsis, and shock are scarce [35]. Surgical debridement is the last resort for aggressive infections that do not respond adequately to antibiotics (Fig. 4).

\section{Skin necrosis}

Flap compromise due to insufficient perfusion can cause different complications depending on its severity. Epidermolysis is a mild variant, and its natural course is towards spontaneous reepithelization (Fig. 5). However, when necrosis occurs in the skin and subdermal tissue, healing can be a tortuous process. Initially, necrosis may manifest with signs of insufficient irrigation, such as delayed capillary fill and diminished local temperature.

The incidence of skin necrosis varies between $3 \%$ and $4.4 \%$ if a limited dissection technique is used, preserving an adequate

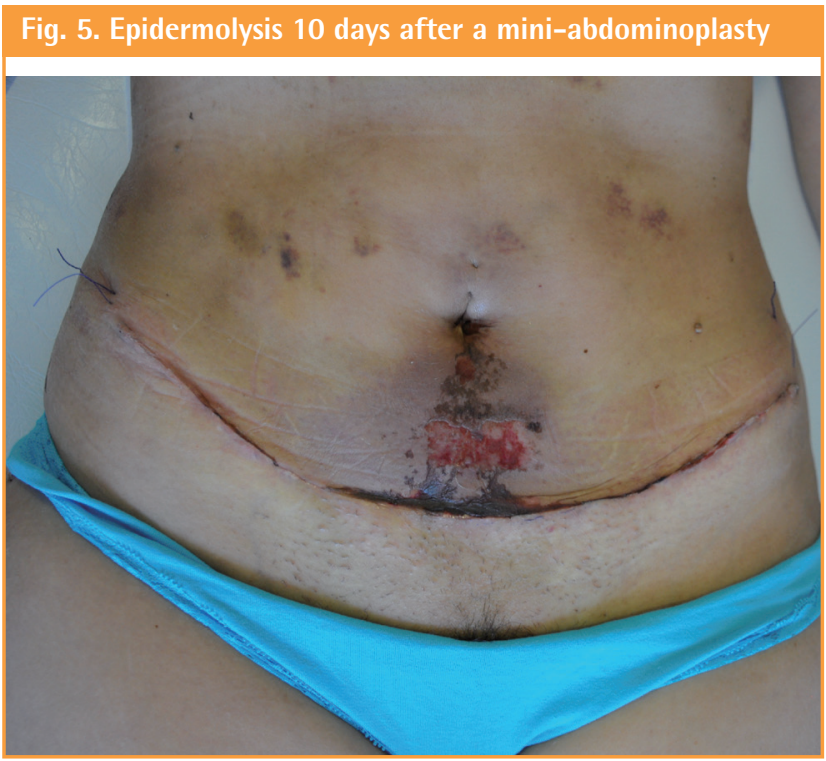

\section{Fig. 4. Surgical debridement of an infected seroma}

$(A, B)$ Preoperative and intraoperative photographs of a 51-yearold female patient who, 1 week after undergoing an abdominoplasty, presented with fever and systemic compromise, erythema in the lower abdomen, and suppuration through the umbilicus incision. She was admitted for antibiotic treatment and surgical debridement. (A) Preoperative photograph; erythema and epidermolysis are noted. $(B, C)$ Intraoperative photographs before (B) and after (C) debridement below the abdominal flap.

number of perforating vessels $[9,11]$. The rate of reoperations to achieve an acceptable aesthetic result associated with this complication is less than $1 \%$.

The most important risk factor for this complication is tobacco consumption, which triples the risk (Level of Evidence: Prognosis, IV [27]). Cessation of this habit lowers the risk and should be encouraged in every patient before surgery. Performing abdominoplasties along with other aesthetic operations at the same time also increases the risk of skin necrosis.

In the great majority of cases, spontaneous evolution leads to healing by secondary intention. Depending on the size of the affected area, this process can take weeks or months (Fig. 6). Close follow-up is essential throughout this period, not only for debridement and dressing of the wound, but also to provide emotional support. A healthy doctor-patient relationship ensures that indications will be followed, and reduces anxiety in patients and their relatives. It is worth mentioning that this is a delicate situation associated with a higher probability of legal repercussions [9].
The use of hyperbaric oxygenation is an adjuvant therapy has been proposed to accelerate the healing process in patients who experience necrosis [36]. Not only does hyperbaric oxygenation increase oxygen availability to different territories by increasing the partial pressure of $\mathrm{O}_{2}$, it also stimulates neovascularization, collagen production, fibroblast proliferation, and mobilization of stem cells from the bone marrow to the injured site $[37,38]$.

The use of negative pressure wound therapy (NPWT) as an adjuvant therapy has also been found to stimulate wound neovascularization and collagen deposition in animal models [39]. NPWT has proven itself useful in a variety of lesions, especially sternotomy wound dehiscence [40] and diabetic foot lesions [41]. However, there is no supporting evidence regarding acute operative wounds, and no benefit has been described for NPWT when used in areas that have been closed by primary intention [42].

\section{Fig. 6. Abdominal flap necrosis}

$(A, B)$ Preoperative photographs of a 64-year-old patient before undergoing an abdominoplasty. (C) Necrotic plaque in the lower end of the abdominal flap 2 weeks after abdominoplasty. (D) Abdominal wound after debridement, 3 weeks after the operation and before starting negative pressure wound therapy (NPWT). (E) The same wound after 2 weeks of NPWT; abundant granulation tissue can be appreciated. (F, G, and H) Postoperative photographs, 3 months after the initial abdominoplasty and 1 month after primary closure of the abdominal wound.
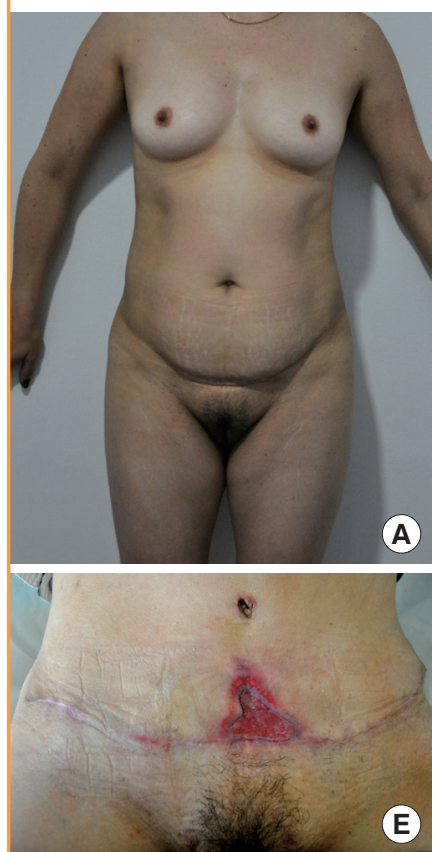
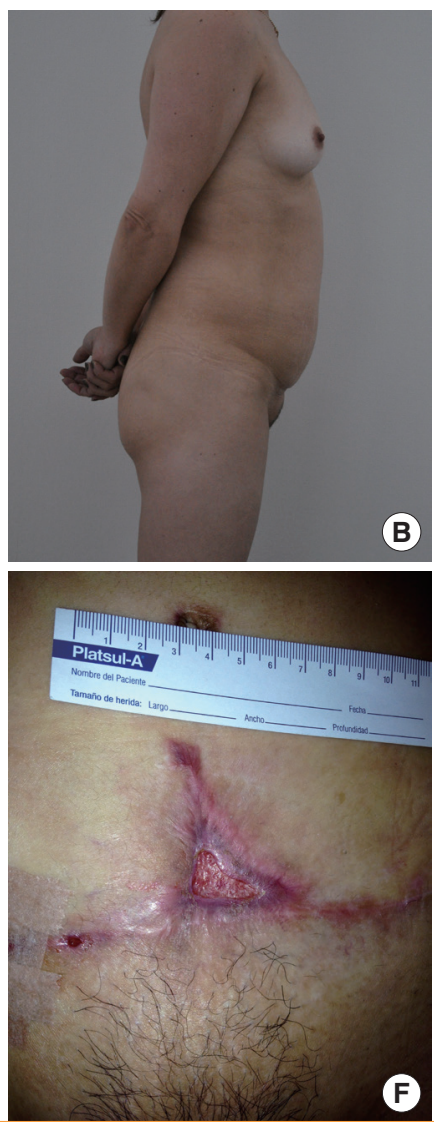
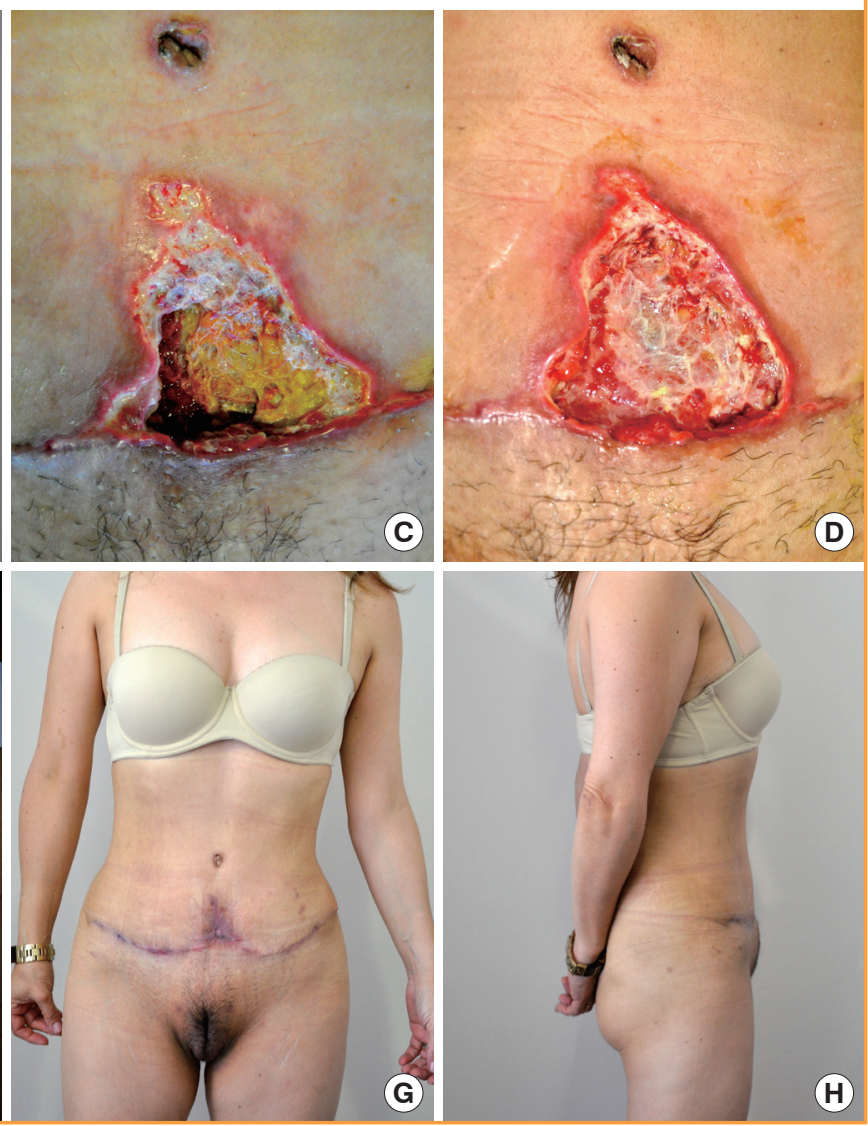


\section{Haematoma}

Haematomas are less frequent than seromas or skin necrosis, with a reported incidence of 2\% [9] Neither Samra et al. [29] nor Hensel et al. [43] encountered any differences in the rate of this complication between patients who underwent abdominoplasties and those who underwent lipoabdominoplasties (Level of Evidence: Prognosis, IV [29]).

The clinical presentation of a haematoma depends on its volume. If it is small enough, it can be completely asymptomatic, but if larger it manifests with swelling, localized pain, and ecchymosis, usually during the first 24 hours. Large haematomas with active bleeding can consequently result in hemodynamic instability and hypovolemic shock, which is a reason why they need to be carefully monitored in order to decide promptly whether exploration is indicated [44].

The risk factors for haematoma as a complication of abdominoplasty, as for any other surgical procedure involving the abdominal wall, are hypertension, unsuccessful haemostasis during the operation, and congenital and acquired coagulopathies. Moreover, a higher incidence of haematoma has been demonstrated in patients with a higher body mass index (Level of Evidence: Prognosis, IV [43]) [25].

The preoperative consultation is the ideal moment to address the patient's past habits and past medical history, focusing on risk factors for bleeding. A previous history of unexplained ecchymosis, haematomas, petechial haemorrhage, or gum bleeding suggest an underlying coagulopathy [45]. Furthermore, the patient must be warned to avoid over-the-counter medications that could alter platelet function during surgery, such as aspirin, non-steroidal anti-inflammatory drugs, phytotherapy, and vita$\min \mathrm{E}$.

The correct treatment of this complication will depend on its volume, time course, the presence of signs and symptoms of bleeding, and hemodynamic status. In the case of a small haematoma, needle aspiration can be attempted to accelerate the recovery process (Fig. 7).

Late-onset haematomas are rare, usually associated with the presence of sutures, and can arise days or weeks after the operation. If the presentation involves acute pain, other causes of abdominal discomfort should be ruled out first [46], and the definitive diagnosis should be confirmed by ultrasound imaging. The surgical team should then decide whether surgical drainage is appropriate depending on the volume and associated symptoms.

\section{Other local complications}

Wound healing after abdominoplasty is affected by the same factors as any other operative wound. A refined surgical tech-

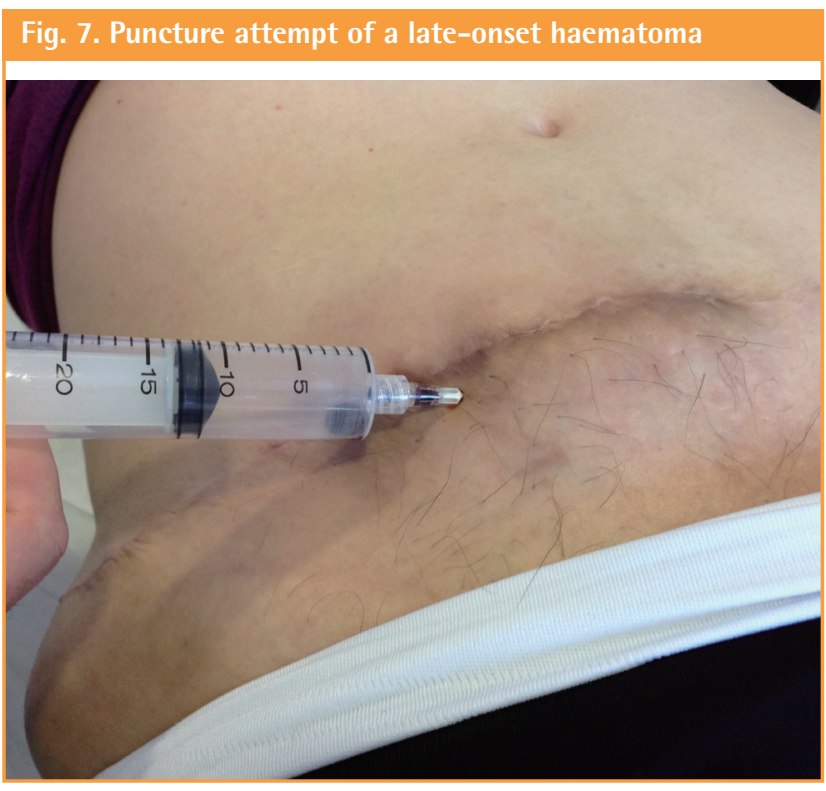

nique should include careful preoperative marking of the area of skin and underlying tissue to be resected to avoid excessive closure tension. During closure, the incision should be sutured in at least 3 planes, which can be aided by the use of barbed sutures to accelerate the closing time by up to $30 \%$ [47-49].

Even with these precautions, the reported incidence of keloid and hypertrophic scars ranges between $1 \%$ and $3.7 \%$. In these cases, compression with silicone strips has proven to be useful (Level of Evidence: Therapeutic, I) [50], leaving intralesional treatments and scar revision as second- and third-line treatments.

Male patients tend to present less pleasing scars than women following abdominoplasty. The inguinal skin in men is thinner and more pigmented than the rest of the skin in the abdominal region. Differences in skin colour between both sides of the scar, along with a disparity in skin thickness, result in a suboptimal aesthetic outcome.

The term "dog ear" is frequently used to describe the conic deformity produced by skin excess after a circular or asymmetrical wound is closed. This defect is always iatrogenic, and in the case of a considerably sized dog ear, it is advisable to correct the defect during the operation, mainly because its postoperative improvement is often unpredictable [51]. A simple alternative for correcting a dog ear is to extend the skin excision in the same direction. It is preferable to perform a $90^{\circ}$ incision at the end of the initial incision and to resect the excess skin or to de-epithelialize the redundant skin, avoiding extending the wound $[52,53]$.

Suture extrusion is another local complication that, according to the published literature, occurs in at least $5 \%$ of cases [11]. The consequent inflammation and swelling usually cause great concern, especially when associated with exudate. Fortunately, 


\section{Fig. 8. Hypertrophic scarring 2 months postoperative}

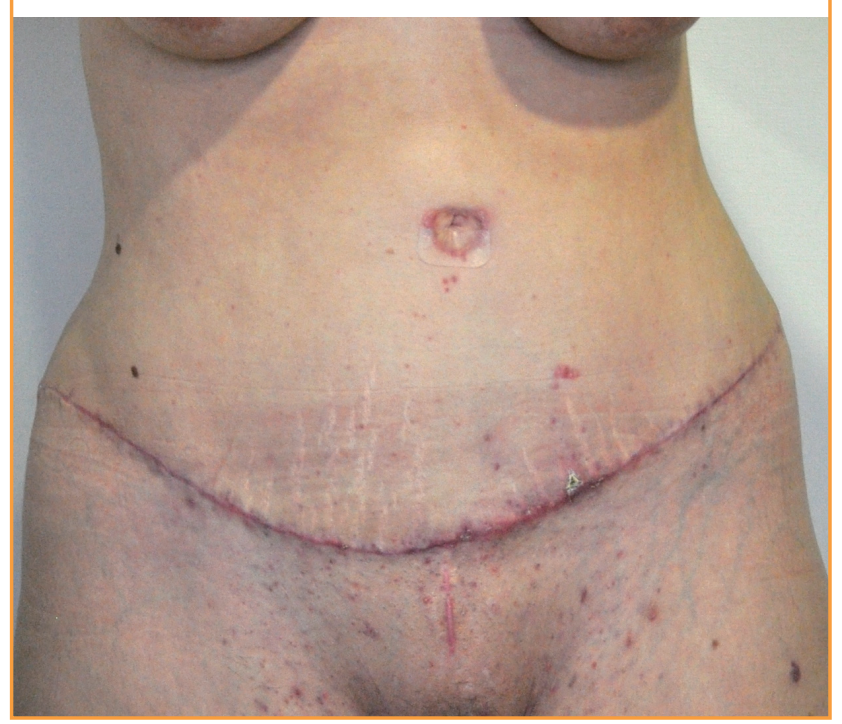

resolution is fast once the offending suture is removed. The use of slow-reabsorption barbed sutures (V-Loc 180, Covidien, Mansfield, MA, USA) is associated with higher rates of suture extrusion, while their fast-absorption variants (V-Loc 90, Covidien) are less closely associated with this side effect (Level of Evidence: Therapeutic, III) [54].

Necrosis of the umbilicus due to insufficient irrigation through its pedicle occurs in about $0.2 \%$ of cases [11]. Special care while performing the plication must be taken to avoid strangulation of the umbilicus.

Several techniques for repositioning the umbilicus have been described, with the goal of obtaining a natural result with a vertical and concave structure [55]. However, there is neither a consensus on where the umbilicus should be placed, nor on the best way to do it [13]. An inverted $\mathrm{V}$ incision in the abdominal flap is the most common technique, according to the published literature, and achieves high satisfaction [56]. It is very important not to perform too large or too small an incision to avoid a redundant or punctiform umbilicus, respectively (Fig. 8).

Approximately $1.9 \%$ of patients suffer some degree of neurological symptoms following abdominoplasty, with the lateral femoral cutaneous nerve the most commonly involved, followed by the iliohypogastric nerve [57]. A careful dissection around the anterior superior iliac spine allows preservation of the lateral femoral cutaneous nerve. Neurological lesions can cause neuropathic pain, hypoesthesia, paraesthesia, hyperesthesia, or allodynia. Meralgia paresthetica is the most common presentation following a lateral cutaneous nerve injury. Once diagnosed, conservative treatment includes massages and analgesia. The use of anticonvulsants, tricyclic antidepressants, nerve blocks, and steroid injections can also be indicated, depending on the clinical presentation. If after 6 months there is no resolution and symptoms are severe, referral to a pain specialist is recommended. Surgical exploration of the nerve trajectory may be useful to free an entrapment or to excise a neuroma $[58,59]$.

Rupture of the vertical plication of the rectus abdominis sheath has been reported. It can occur years after the abdominoplasty and present as fast-growing abdominal pseudo-tumours [60]. Clinically, these are difficult to differentiate from malignant tumours or hernias, and for this reason, imaging studies are usually required to confirm the diagnosis.

\section{Systemic complications}

Systemic complications are the most feared and severe complications and, fortunately, the least frequent ones after an abdominoplasty. The incidence of thromboembolism ranges between $0.3 \%$ and $1.1 \%$, depending on the series $[9,11]$. These reports contain cases diagnosed using Doppler ultrasound imaging, without consideration of subclinical deep venous thromboses that resolve spontaneously without causing symptoms. Apart from the classically described risk factors for this complication, patients undergoing abdominoplasty are at an even higher risk if their body mass index is $30 \mathrm{~kg} / \mathrm{m}^{2}$ or more (Level of Evidence: Prognosis, IV) [61]. Combining this surgery with other intraabdominal operations at the same time increases the risk of deep venous thrombosis to $2.17 \%$, contrasting with an incidence of $0.76 \%$ when it is associated with another aesthetic procedure [61]. Reports of fat embolism following an abdominoplasty are scarce [62].

The indication for thromboembolism prophylaxis in abdominoplasties is still a controversial issue. Newall et al. [63] and Hatef et al. [64] have reported that using low-molecular-weight heparin in high-risk patients reduced the rate of deep venous thrombosis (Level of Evidence: Prognosis, I). These studies also showed a consequent increase in the incidence of haematomas when chemoprophylaxis was used. Other authors prefer prevention protocols that avoid the use of low-molecular-weight heparin by using intermittent pneumatic compression intraoperatively and until the patient is discharged, in combination with early assisted walking in the first postoperative hours. These precautions, along with ensuring tobacco cessation for at least a month before surgery, allowed Somogyi et al. [65] to report only 1 case of deep venous thrombosis among the 404 patients in their case series (Level of Evidence: Therapeutic, IV).

For years, it was thought that abdominoplasty could produce respiratory insufficiency secondary to the plication of the rectus abdominis sheath. To investigate this potential association, Tercan et al. [66] measured preoperative respiratory function before an abdominoplasty and repeated the tests 10 and 30 days 
after the operation. Forced expiratory volume in 1 second was the same at all 3 checkpoints. Interestingly, the forced vital capacity was reduced in $4 \%$ of patients 10 days after the abdominoplasty, recovering to its preoperative level 30 days postoperatively. However, anecdotal cases have been reported of respiratory distress caused by excessive tension.

Death following an abdominoplasty has rarely been reported in the literature [67], with an incidence ranging from $0.04 \%$ to $0.16 \%$ in series published around 25 years ago $[68,69]$, and mortality has not been mentioned in more recent series. Most cases of mortality were attributed to massive pulmonary embolism. However, these statistics do not consider abdominoplasties performed by uncertified plastic surgeons working under limited safety conditions.

\section{DISCUSSION}

This literature review intended to systematically present the available evidence regarding the incidence, prevention, and treatment of the most common complications following abdominoplasty. Even though a more exhaustive methodological scrutiny of the articles referenced in this review would have been desirable, doing so would have meant focusing on specific aspects of abdominoplasty complications, rather than providing a comprehensive overview. For this reason, the authors limited themselves to mentioning the design of each study, along with the according level of evidence, for the studies cited in this article. A statistical meta-analysis of the results exposed is beyond the scope of this study, mainly due to the heterogeneity of the studies involved.

Another limitation worth mentioning is the fact that 9 out of 119 studies were excluded for being published in languages not intelligible to the authors. The remaining 110 articles were mostly in English, with only 1 published in Spanish. In an ideal future review, language should not be part of the inclusion criteria. For the same reason, it would be interesting to include other indexing databases in the systematic search strategy, especially those that contain un-published results. This is particularly relevant because complications may be prone to under-reporting in the scientific literature.

As was presented in the results section, abdominoplasty, with or without liposuction, is a safe procedure when adequate precautions are taken before, during, and after surgery. Patient preparation is of the foremost importance. It is during the initial consultation that tobacco cessation can be addressed, potentially reducing the risk of infection and skin necrosis. In a similar manner, there is an opportunity to diagnose and treat patients who present with iron-deficiency anaemia. Up to $10 \%$ women of child-bearing age have haemoglobin concentrations below 12 $\mathrm{g} / \mathrm{dL}[70]$.

During the operation, a polished surgical technique is essential, informed by the latest evidence and the individual surgeon's experience. The search for improved aesthetic outcomes and reduced complication rates should motivate every specialist to polish his or her abilities and to make corrections when needed. For that matter, in the past decades it has been proven that adding liposuction to an abdominoplasty does not put patients at additional risk, as long as perfusion of the flap is secured by preserving enough perforators through limited dissection. Regarding seroma prevention, progressive tension sutures, the use of fibrin sealant, and dissection over the Scarpa fascia have been proposed as well. However, the adoption of these strategies has not been widespread, possibly due to the observer bias present in the studies supporting these interventions.

The mini-abdominoplasty technique is an alternative for a number of patients who do not require a large skin and fat excision and want to avoid a longer scar [71]. Even though the complication rates are similar to those of traditional abdominoplasties, there are some specific considerations regarding the aesthetic results of this technique. Dog ear deformities are common with this technique and usually require scar elongation. Furthermore, the most critiqued aspect of mini-abdominoplasties is related to the appearance of the umbilicus. Once the umbilical pedicle is cut, it loses its normal anchor and remains attached to the surrounding skin [72]. This inevitably produces a change in the position of the umbilicus in the caudal direction, depending on the amount of skin that is excised. Once the abdominal flap is sutured under tension, the umbilicus is deformed, adopting a thin vertical form. Additionally, this technique presents a limitation if a secondary abdominoplasty is indicated in the future, because it is impossible to reposition the umbilicus after dividing its pedicle. The only remaining solution in such cases is to recreate a neo-umbilicus, which often has a suboptimal appearance.

Operative time is another factor that has recently received special attention in plastic surgery, considering the duration of some procedures and the possibility of combining multiple procedures at the same time. In a retrospective review of 1,753 plastic surgery procedures, operative time was associated with higher complication rates (Level of Evidence: Prognosis, IV) [73]. Particularly, interventions of 4 hours or more were significantly associated with higher postoperative morbidity, with an odds ratio (OR) of 1.61, considering infections, wound dehiscence, flap necrosis, seromas, and haematomas. The risk was even higher for procedures lasting more than 5 hours and more than 7 hours, increasing the OR to 3.05 and 4.71 , respectively. The 
same conclusion was obtained after the sample was adjusted according to surgical complexity.

Postoperative consultations present an opportunity to diagnose complications, even though they might be asymptomatic at certain points of the recovery process. Maintaining a good pa- tient-doctor relationship is the key for treating any complication while maintaining the patient's trust. Complications not only affect medical recovery, but also harm expectations.

Inevitably, there will be a small group of patients who will not be satisfied with the final cosmetic result. Sometimes, this will

\section{Fig. 9. Secondary abdominoplasty}

$(A, B)$ Preoperative photographs of a 61-year-old female patient with a previous history of diabetes and tobacco consumption who had undergone an abdominoplasty eight years before. (C, D) Postoperative photographs 3 months after a secondary abdominoplasty. The patient chose a scar in a cephalic position rather than a vertical scar caused by repositioning the umbilicus.
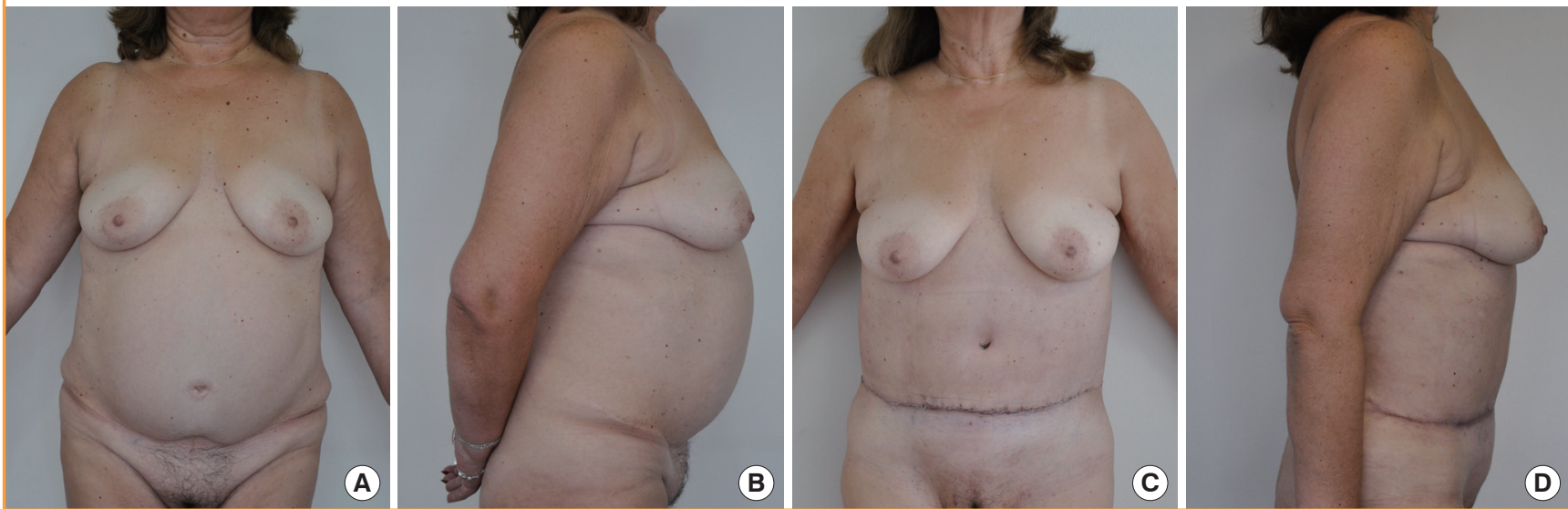

Fig. 10. Non-complicated abdominoplasty case

(A-F) Preoperative and 3-month postoperative photographs of a 41-year-old post-bariatric female patient who underwent a lipoabdominoplasty with a simultaneous augmentation mastopexy with silicone implants.
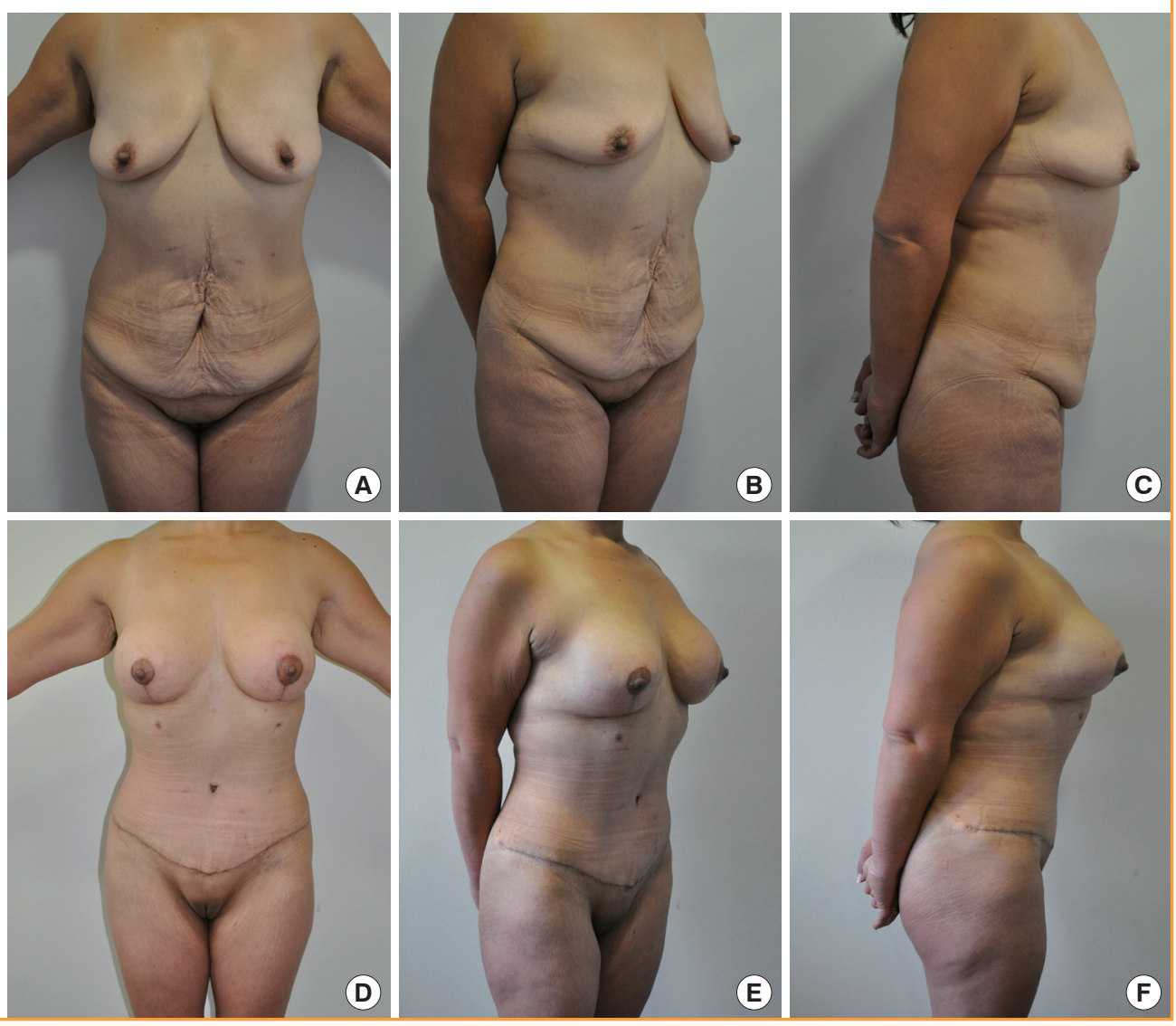
be caused by surgical difficulties or by the emergence of a complication that compromises the aesthetic outcome; occasionally, preoperative patient expectations may simply have been too high to be satisfied $[74,75]$.

There is an even smaller group of patients who will require a secondary abdominoplasty to improve a suboptimal result; this procedure can be carried out by the same surgeon that performed the first operation or by another surgeon. It is worth mentioning that these operations have a higher risk of complications and usually exhibit a slower recovery. Despite this, there is no reason to give up when facing these challenges, because the results can be impressive (Fig. 9) [76].

Despite all the complications mentioned in this article, abdominoplasties and lipoabdominoplasties are reproducible and very gratifying operations for both patients and surgeons. This is the result of a long process that has involved numerous authors who have shared their experiences, not only including successful cases, but also their most challenging complications (Fig. 10).

\section{PATIENT CONSENT}

The patient provided written informed consent for the publication and the use of their images.

\section{REFERENCES}

1. International Society of Aesthetic Plastic Surgery. International study on aesthetic/cosmetic procedures performed in 2013. Hanover: International Society of Aesthetic Plastic Surgery; 2014.

2. Kelly HA. Excision of the fat of the abdominal wall lipectomy. Surg Gynecol Obstet 1910;10:229-31.

3. Vernon S. Umbilical transplantation upward and abdominal contouring in lipectomy. Am J Surg 1957;94:490-2.

4. Pitanguy I. Abdominal lipectomy: an approach to it through an analysis of 300 consecutive cases. Plast Reconstr Surg 1967;40:384-91.

5. Grazer FM. Abdominoplasty. Plast Reconstr Surg 1973;51: 617-23.

6. Ousterhout DK. Combined suction-assisted lipectomy, surgical lipectomy, and surgical abdominoplasty. Ann Plast Surg 1990;24:126-32.

7. Swanson E. Prospective outcome study of 360 patients treated with liposuction, lipoabdominoplasty, and abdominoplasty. Plast Reconstr Surg 2012;129:965-78.

8. Saldanha OR, Federico R, Daher PF, et al. Lipoabdominoplasty. Plast Reconstr Surg 2009;124:934-42.

9. Matarasso A, Swift RW, Rankin M. Abdominoplasty and abdominal contour surgery: a national plastic surgery survey. Plast Reconstr Surg 2006;117:1797-808.

10. OCEBM Levels of Evidence Working Group. The Oxford levels of evidence 2. Oxford Center Evidence-Based Medicine [Internet]. Oxford; Centre for Evidence-Based Medicine; 2011 [cited 2017 Jun 5]. Available from: http://www. cebm.net/index.aspx?o $=5653$.

11. Neaman KC, Armstrong SD, Baca ME, et al. Outcomes of traditional cosmetic abdominoplasty in a community setting: a retrospective analysis of 1008 patients. Plast Reconstr Surg 2013;131:403e-410e.

12. Najera RM, Asheld W, Sayeed SM, et al. Comparison of seroma formation following abdominoplasty with or without liposuction. Plast Reconstr Surg 2011;127:417-22.

13. Hurvitz KA, Olaya WA, Nguyen A, et al. Evidence-based medicine: abdominoplasty. Plast Reconstr Surg 2014;133: 1214-21.

14. Costa-Ferreira A, Rebelo M, Silva A, et al. Scarpa fascia preservation during abdominoplasty: randomized clinical study of efficacy and safety. Plast Reconstr Surg 2013;131:644-51.

15. Fang RC, Lin SJ, Mustoe TA. Abdominoplasty flap elevation in a more superficial plane: decreasing the need for drains. Plast Reconstr Surg 2010;125:677-82.

16. Swanson E. Scarpa fascia preservation during abdominoplasty: randomized clinical study of efficacy and safety. Plast Reconstr Surg 2013;132:871e-873e.

17. Marsh DJ, Fox A, Grobbelaar AO, et al. Abdominoplasty and seroma: a prospective randomised study comparing scalpel and handheld electrocautery dissection. J Plast Reconstr Aesthet Surg 2015;68:192-6.

18. Baroudi R, Ferreira CA. Seroma: how to avoid it and how to treat it. Aesthet Surg J 1998;18:439-41.

19. Pollock TA, Pollock H. Progressive tension sutures in abdominoplasty: a review of 597 consecutive cases. Aesthet Surg J 2012;32:729-42.

20. Pilone V, Vitiello A, Borriello C, et al. The use of a fibrin glue with a low concentration of thrombin decreases seroma formation in postbariatric patients undergoing circular abdominoplasty. Obes Surg 2015;25:354-9.

21. Lee JC, Teitelbaum J, Shajan JK, et al. The effect of fibrin sealant on the prevention of seroma formation after postbariatric abdominoplasty. Can J Plast Surg 2012;20:178-80.

22. Throckmorton AD, Askegard-Giesmann J, Hoskin TL, et al. Sclerotherapy for the treatment of postmastectomy seroma. Am J Surg 2008;196:541-4.

23. Roje Z, Karanovic N, Utrobicic I. Abdominoplasty complications: a comprehensive approach for the treatment of chronic seroma with pseudobursa. Aesthetic Plast Surg 
2006;30:611-5.

24. Nayak N, Narayan D. Buried dermal flap for the treatment of chronic postoperative seroma. BMJ Case Rep 2010;2010: bcr0120102668.

25. Rogliani M, Silvi E, Labardi L, et al. Obese and nonobese patients: complications of abdominoplasty. Ann Plast Surg 2006;57:336-8.

26. Vastine VL, Morgan RF, Williams GS, et al. Wound complications of abdominoplasty in obese patients. Ann Plast Surg 1999;42:34-9.

27. Manassa EH, Hertl CH, Olbrisch RR. Wound healing problems in smokers and nonsmokers after 132 abdominoplasties. Plast Reconstr Surg 2003;111:2082-7.

28. Hopf HW, Rollins MD. Wounds: an overview of the role of oxygen. Antioxid Redox Signal 2007;9:1183-92.

29. Samra S, Sawh-Martinez R, Barry O, et al. Complication rates of lipoabdominoplasty versus traditional abdominoplasty in high-risk patients. Plast Reconstr Surg 2010;125: 683-90.

30. Fry DE, Barie PS. The changing face of Staphylococcus aureus: a continuing surgical challenge. Surg Infect (Larchmt) 2011;12:191-203.

31. Meyers H, Brown-Elliott BA, Moore D, et al. An outbreak of Mycobacterium chelonae infection following liposuction. Clin Infect Dis 2002;34:1500-7.

32. Murillo J, Torres J, Bofill L, et al. Skin and wound infection by rapidly growing mycobacteria: an unexpected complication of liposuction and liposculpture. The Venezuelan Collaborative Infectious and Tropical Diseases Study Group. Arch Dermatol 2000;136:1347-52.

33. Sevin A, Senen D, Sevin K, et al. Antibiotic use in abdominoplasty: prospective analysis of 207 cases. J Plast Reconstr Aesthet Surg 2007;60:379-82.

34. Casaer B, Tan EK, Depoorter M. The role of antibiotic prophylaxis in abdominoplasty: a review of the infection rate in 300 cases treated without prophylaxis. Plast Reconstr Surg 2009; 123:42e.

35. Gaede FM, Ouazzani A, de Fontaine S. Necrotizing fasciitis after abdominoplasty. Plast Reconstr Surg 2008;121:358-9.

36. Berner JE, Vidal P, Will P, et al. Use of hyperbaric oxygenation for wound management. Rev Med Chil 2014;142: 1575-83.

37. Thom SR. Hyperbaric oxygen: its mechanisms and efficacy. Plast Reconstr Surg 2011;127 Suppl 1:131S-141S.

38. Howard MA, Asmis R, Evans KK, et al. Oxygen and wound care: a review of current therapeutic modalities and future direction. Wound Repair Regen 2013;21:503-11.

39. Wang W, Pan Z, Hu X, et al. Vacuum-assisted closure in- creases ICAM-1, MIF, VEGF and collagen I expression in wound therapy. Exp Ther Med 2014;7:1221-6.

40. Damiani G, Pinnarelli L, Sommella L, et al. Vacuum-assisted closure therapy for patients with infected sternal wounds: a meta-analysis of current evidence. J Plast Reconstr Aesthet Surg 2011;64:1119-23.

41. Guffanti A. Negative pressure wound therapy in the treatment of diabetic foot ulcers: a systematic review of the literature. J Wound Ostomy Continence Nurs 2014;41:233-7.

42. Webster J, Scuffham P, Sherriff KL, et al. Negative pressure wound therapy for skin grafts and surgical wounds healing by primary intention. Cochrane Database Syst Rev 2012; (4):CD009261.

43. Hensel JM, Lehman JA Jr, Tantri MP, et al. An outcomes analysis and satisfaction survey of 199 consecutive abdominoplasties. Ann Plast Surg 2001;46:357-63.

44. David JS, Spann C, Marcotte G, et al. Haemorrhagic shock, therapeutic management. Ann Fr Anesth Reanim 2013;32: 497-503.

45. Ballas M, Kraut EH. Bleeding and bruising: a diagnostic work-up. Am Fam Physician 2008;77:1117-24.

46. da Freitas RS, Ascenco AS, Maluf Junior I, et al. Acute abdomen after abdominoplasty: differential diagnosis. Aesthetic Plast Surg 2013;37:1182-5.

47. Gutowski KA, Warner JP. Incorporating barbed sutures in abdominoplasty. Aesthet Surg J 2013;33(3 Suppl):76S-81S.

48. Moya AP. Barbed sutures in body surgery. Aesthet Surg J 2013;33(3 Suppl):57S-71S.

49. Shermak MA. The application of barbed sutures in body contouring surgery. Aesthet Surg J 2013;33(3 Suppl):72S$75 S$.

50. O’Brien L, Jones DJ. Silicone gel sheeting for preventing and treating hypertrophic and keloid scars. Cochrane Database Syst Rev 2013;(9):CD003826.

51. Jaibaji M, Morton JD, Green AR. Dog ear: an overview of causes and treatment. Ann R Coll Surg Engl 2001;83:136-8.

52. Grassetti L, Lazzeri D, Torresetti M, et al. Aesthetic refinement of the dog ear correction: the 90 degrees incision technique and review of the literature. Arch Plast Surg 2013;40: 268-9.

53. Loh CY, Loh AY, Mashhadi SA. A novel method for repairing the dog ear. J Plast Reconstr Aesthet Surg 2013;66:e341-2.

54. Rubin JP, Hunstad JP, Polynice A, et al. A multicenter randomized controlled trial comparing absorbable barbed sutures versus conventional absorbable sutures for dermal closure in open surgical procedures. Aesthet Surg J 2014;34: 272-83.

55. Craig SB, Faller MS, Puckett CL. In search of the ideal fe- 
male umbilicus. Plast Reconstr Surg 2000;105:389-92.

56. Malic CC, Spyrou GE, Hough M, et al. Patient satisfaction with two different methods of umbilicoplasty. Plast Reconstr Surg 2007;119:357-61.

57. Ducic I, Zakaria HM, Felder JM 3rd, et al. Abdominoplastyrelated nerve injuries: systematic review and treatment options. Aesthet Surg J 2014;34:284-97.

58. Lee CH, Dellon AL. Surgical management of groin pain of neural origin. J Am Coll Surg 2000;191:137-42.

59. Madura JA, Madura JA 2nd, Copper CM, et al. Inguinal neurectomy for inguinal nerve entrapment: an experience with 100 patients. Am J Surg 2005;189:283-7.

60. Dragu A, Bach AD, Polykandriotis E, et al. Pseudotumors after primary abdominal lipectomy as a new sequela in patients with abdominal apron. Obes Surg 2009; 19:1599-604.

61. Hatef DA, Kenkel JM, Nguyen MQ, et al. Thromboembolic risk assessment and the efficacy of enoxaparin prophylaxis in excisional body contouring surgery. Plast Reconstr Surg 2008;122:269-79.

62. Shaikh N, Hanssens Y, Kettern MA, et al. Cerebral fat embolism as a rare complication of liposuction with abdominoplasty. Rev Neurol 2008;47:277-8.

63. Newall G, Ruiz-Razura A, Mentz HA, et al. A retrospective study on the use of a low-molecular-weight heparin for thromboembolism prophylaxis in large-volume liposuction and body contouring procedures. Aesthetic Plast Surg 2006; 30:86-95.

64. Hatef DA, Trussler AP, Kenkel JM. Procedural risk for venous thromboembolism in abdominal contouring surgery: a systematic review of the literature. Plast Reconstr Surg 2010;125:352-62.

65. Somogyi RB, Ahmad J, Shih JG, et al. Venous thromboembolism in abdominoplasty: a comprehensive approach to lower procedural risk. Aesthet Surg J 2012;32:322-9.

66. Tercan M, Bekerecioglu M, Dikensoy O, et al. Effects of ab- dominoplasty on respiratory functions: a prospective study. Ann Plast Surg 2002;49:617-20.

67. Christman KD. Death following suction lipectomy and abdominoplasty. Plast Reconstr Surg 1986;78:428.

68. Grazer FM, Goldwyn RM. Abdominoplasty assessed by survey, with emphasis on complications. Plast Reconstr Surg 1977;59:513-7.

69. Teimourian B, Rogers WB 3rd. A national survey of complications associated with suction lipectomy: a comparative study. Plast Reconstr Surg 1989;84:628-31.

70. Rios-Castillo I, Brito A, Olivares M, et al. Low prevalence of iron deficiency anemia between 1981 and 2010 in Chilean women of childbearing age. Salud Publica Mex 2013;55: 478-83.

71. Sim HB, Yoon SY. Experiences of abdominoplasty without undermining. J Korean Soc Plast Reconstr Surg 2006;33: 303-7.

72. Cido Carvalho FAM, Vieira da Silva VJr, Alencar Moreira A. Lipoabdominoplasty with umbilical detachment and reduced abdominal undermining. Cir Plast Iberolatinoam 2008;34:277-85.

73. Hardy KL, Davis KE, Constantine RS, et al. The impact of operative time on complications after plastic surgery: a multivariate regression analysis of 1753 cases. Aesthet Surg J 2014;34:614-22.

74. Flynn TC, Narins RS. Preoperative evaluation of the liposuction patient. Dermatol Clin 1999; 17:729-34.

75. Rochefort G, Berner JE, Castillo P, et al. The importance of diagnosing body dysmorphic disorder in the Aesthetic Surgery Consultation. Cir Plast Iberolatinoam 2014;40:253-9.

76. Matarasso A, Schneider LF, Barr J. The incidence and management of secondary abdominoplasty and secondary abdominal contour surgery. Plast Reconstr Surg 2014;133:4050. 